\title{
Class 1, 2, and 3 BRAF-Mutated Metastatic Colorectal Cancer: A Detailed Clinical, Pathologic, and Molecular Characterization
}

\author{
Marta Schirripa', Paola Biason', Sara Lonardi ${ }^{1}$, Nicoletta Pella ${ }^{2}$, Maria Simona Pino ${ }^{3}$, \\ Federica Urbano ${ }^{4}$, Carlotta Antoniotti ${ }^{5}$, Chiara Cremolini ${ }^{5}$, Salvatore Corallo ${ }^{6}$, \\ Filippo Pietrantonio ${ }^{6,7}$, Fabio Gelsomino $^{8}$, Stefano Cascinu ${ }^{8}$, Armando Orlandi ${ }^{9}$, \\ Giada Munari ${ }^{1,10}$, Umberto Malapelle ${ }^{11}$, Serena Saggio ${ }^{6}$, Gabriella Fontanini ${ }^{12}$, \\ Massimo Rugge ${ }^{10}$, Claudia Mescoli ${ }^{10}$, Stefano Lazzi ${ }^{13}$, Luca Reggiani Bonetti ${ }^{14}$, \\ Giovanni Lanza ${ }^{15}$, Angelo Paolo Dei Tos ${ }^{16}$, Giovanna De Maglio ${ }^{17}$, Maurizio Martini ${ }^{18,19}$ \\ Francesca Bergamo ${ }^{1}$, Vittorina Zagonel', Fotios Loupakis ${ }^{1}$, and Matteo Fassan ${ }^{10}$
}

\begin{abstract}
Purpose: BRAF mutations are grouped in activating RASindependent signaling as monomers (class $1-\mathrm{V} 600 \mathrm{E}$ ) or as dimers (class 2-codons 597/601), and RAS-dependent with impaired kinase activity (class 3-codons 594/596). Although clinical, pathologic, and molecular features of ${ }^{V 600 E} B R A F-$ mutated metastatic colorectal cancer (mCRC) are well known, limited data are available from the two other classes.

Experimental Design: Data from 117 patients with $B R A F$ (92 class 1,12 class 2 , and 13 class 3 )-mutated mCRC were collected. A total of 540 BRAF wt mCRCs were included as control. IHC profiling was performed to determine the consensus molecular subtypes (CMS), cytokeratin 7/20 profiles, tumor-infiltrating lymphocyte infiltration, and BM1/BM2 categorization. Overall survival (OS) and progression-free survival were evaluated by Kaplan-Meier and log-rank test.
\end{abstract}

Results: Class 3 BRAF-mutated mCRC was more frequently left sided $(P=0.0028)$, pNO $(P=0.0159)$, and without peritoneal metastases $(P=0.0176)$ compared with class 1 , whereas class 2 cases were similar to class 1 . Hazard ratio for OS, as compared with BRAF wt, was 2.38 [95\% confidence interval (CI), 1.61-3.54] for class 1, 1.90 (95\% CI, 0.85-4.26) for class 2, and 0.93 (95\% CI, 0.51-1.69) for class 3 $(P<0.0001)$. Class 2 and 3 tumors were all assigned to CMS2-3. A higher median CD3/CD8-positive lymphocyte infiltration was observed in BRAF-mutated class $2(P=$ 0.033 ) compared with class 3 cases.

Conclusions: For the first time, different clinical and pathologic features and outcome data were reported according to the three BRAF mutation classes in mCRC. Specific targeted treatment strategies should be identified in the near future for such patients.

\section{Introduction}

Analysis of molecular alteration like $R A S,{ }^{V 600 E} B R A F$ mutation, and microsatellite instability are nowadays a consolidated routine in the assessment of patients with metastatic colorectal cancer (mCRC) (1) because all of them have a clear prognostic and/or predictive role.
Department of Oncology, Veneto Institute of Oncology IOV - IRCCS, Padua, Italy. ${ }^{2}$ Department of Oncology, University and General Hospital, Udine, Italy. ${ }^{3}$ Medical Oncology Unit, Department of Oncology, Azienda USL Toscana Centro, S. Maria Annunziata Hospital, Florence, Italy. ${ }^{4}$ Department of Radiological Sciences, Oncology and Pathology, Policlinico Umberto I, Sapienza University of Rome, Rome, Italy. ${ }^{5}$ Unit of Medical Oncology, Department of Translational Research and New Technologies in Medicine and Surgery, University of Pisa, Pisa, Italy. ${ }^{6}$ Department of Medical Oncology, Fondazione IRCCS Istituto Nazionale dei Tumori, Milan, Italy. ${ }^{7}$ Department of Oncology and Hemato-oncology, University of Milan, Milan, Italy. ${ }^{8}$ Department of Oncology and Haematology, University Hospital of Modena, Modena, Italy. ${ }^{9}$ U.O.C Oncologia, Fondazione Policlinico Universitario Agostino Gemelli IRCCS, Rome, Italy. ${ }^{10}$ Surgical Pathology and Cytopathology Unit, Department of Medicine (DIMED), University of Padua, Padua, Italy. "Department of Public Health, University Federico II of Naples, Naples, Italy. ${ }^{12}$ Department of Surgical, Medical, Molecular Pathology and Critical Area, University of Pisa, Pisa, Italy. ${ }^{13}$ Department of Medical Biotechnology, Section of Pathology, University of Siena, Siena, Italy. ${ }^{14}$ Department of Diagnostic Medicine and Public Health, Section of Pathology, Università di Modena e Reggio Emilia, Policlinico di Modena, Modena, Italy. ${ }^{15}$ Department of Pathology, University of Ferrara, Ferrara, Italy. ${ }^{16}$ Department of Pathology and
Molecular Genetics, Treviso General Hospital, Treviso, Italy. ${ }^{17}$ Department of Pathology, University Hospital of Udine, Udine, Italy. ${ }^{18}$ Dipartimento Scienze della Salute della Donna, del Bambino e di Sanità Pubblica, area di Anatomia Patologica, Fondazione policlinico Universitario A. Gemelli IRCCS, Rome, Italy. ${ }^{19}$ Istituto di Anatomia Patologica, Università Cattolica Del Sacro Cuore, Rome, Italy.

Note: Supplementary data for this article are available at Clinical Cance Research Online (http://clincancerres.aacrjournals.org/).

M. Schirripa and P. Biason contributed equally as co-first authors of this article. S. Lonardi, F. Loupakis, and M. Fassan contributed equally as senior authors of this article.

Corresponding Author: Fotios Loupakis, Oncologia Medica 1, Istituto Oncologico Veneto IOV - IRCCS, via Gattamelata 64, Padua 35128, Italy. Phone: +39049-8215919; Fax: +39-049-8217929; E-mail: fotios.loupakis@iov.veneto.it

Clin Cancer Res 2019;25:3954-61

doi: 10.1158/1078-0432.CCR-19-0311

(C)2019 American Association for Cancer Research. 


\section{Translational Relevance}

This study focused on two rare and distinct subgroups of non-V600E BRAF-mutated metastatic colorectal cancer patients while comparing them with ${ }^{\mathrm{V} 600 \mathrm{E}} B R A F$-mutated and a control set of wild-type patients. A detailed description of clinical and pathologic features, including consensus molecular subtypes and BM1/BM2 categorization according to Barras and colleagues, is reported along with outcome data. Results underline the importance of ${ }^{\text {non- } V 600 \mathrm{E}}$ BRAF mutation detection and point out the need for focusing therapeutic research efforts, taking into account the specificities of these peculiar colorectal cancer subtypes.

Patients with ${ }^{V 600 E} B R A F$-mutated mCRC share specific clinical and pathologic features, such as older age at diagnosis, female sex, right sided location of primary tumors, poor differentiation, mucinous histology, and microsatellite instability (2-4). In the metastatic setting, ${ }^{V O O O E} B R A F$ mutation occurs in approximately $10 \%$ of cases, and it is associated with poor prognosis and scarce overall benefit from standard therapeutic approaches $(5,6)$. Recently, specific gene expression profiles were described for distinguishing two subgroups among ${ }^{V 600 E} B R A F$-mutated cancers, named BM1 (showing activation of KRAS/mTOR/AKT/ 4EBP1 pathway) and BM2 (with deregulation in the cell cycle; ref. 7).

In recent years, thanks to the adoption of more accurate techniques for mutational status evaluation such as nextgeneration sequencing and mass spectrometry, other rare BRAF mutations have been identified. Overall ${ }^{\text {non-VGOOE }} B R A F$ mutations occur in only $2 \%$ of patients with mCRC and cover 19 different codons $(8,9)$. The clinical significance of these mutations is largely unknown, due to the rarity of this condition.

Intriguing data on clinical features of ${ }^{\text {non-VGOOE }} B R A F$ mutations in patients with colorectal cancer emerged from two recent retrospective cohort studies. Those reports agree in defining the ${ }^{n O n-V 600 E} B R A F$-mutated population (mainly including mutations in codons 594 and 596) as a distinct subgroup with its own features, in particular longer overall survival (OS) compared with ${ }^{V 600 E} B R A F$-mutated patients, no association with older age, female sex, right sided tumor, mucinous histology, peritoneal spread, and microsatellite instability $(8,10)$.

A deeper knowledge of ${ }^{n o n-V 600 E}$ BRAF mutation derived from functional studies on noncolorectal preclinical models, which led to identify three classes of BRAF mutations: activating RAS-independent BRAF mutations signaling as monomers (class 1) or as dimers (class 2) and RAS-dependent BRAF mutations with impaired kinase activity or kinase-dead (class 3; ref. 9). ${ }^{V 600 E} B R A F$ mutation belongs to class 1 , whereas among non-V600E $B R A F$ mutations those affecting codons 601 and 597 are assigned to class 2; while those in codons 594 and 596 to class 3. Preliminary data, mainly derived from melanoma models, suggested also a different sensitivity to BRAF inhibitors based on the three classes $(5,9,11)$.

Moving from all the above considerations, the simple distinction in V600E and non-V600E mutations may be too simplistic, and a specific characterization of BRAF class 2 and 3 mutations is needed.
This study aimed to provide a specific clinical, pathologic, molecular, and prognostic characterization of patients with ${ }^{n o n-V 6 O 0 E}$ BRAF-mutated mCRC.

\section{Materials and Methods}

\section{Study design}

Patients were categorized as follows: (i) BRAF wild-type, (ii) $B R A F-$ mutant class 1 (i.e. harboring the V600E alteration), (iii) $B R A F$-mutant class 2 (i.e. harboring codons 601 or 597 alterations), and (iv) BRAF-mutant class 3 (i.e., harboring codons 594 or 596 alterations). For all the BRAF-mutated cases, tissue specimens (paraffin embedded block or, as an alternative, $5-\mu \mathrm{m}$-thick slides for IHC analyses) of primary and/or metastases were collected from the archives of the referral Pathology Departments.

Data on availability of colorectal cancer samples diagnosed with ${ }^{\text {non-V60OE }}$ BRAF mutations were firstly retrieved from the 9 Italian Surgical Pathology Units involved in the study. Clinical and survival data of identified patients were retrieved from 8 Italian Oncology Units and matched with available molecular and pathologic information. Main inclusion criteria were: diagnosis of metastatic colorectal cancer and available clinical data. Clinicopathologic and survival data of ${ }^{V 600 E} B R A F-$ mutated and $B R A F$ wild-type cases were collected from patients referred to the Veneto Institute of Oncology, Padua, from January 2010 to December 2016. Clinical and pathologic features are described in Table 1 . The study was approved by the Ethics Committee of Veneto Institute of Oncology and was conducted according to ethical standards laid down in the 1964 Declaration of Helsinki and its later amendments. Written informed consent was obtained from all the patients.

\section{Mutational status analyses}

RAS and BRAF mutational profiling were carried out on formalin-fixed paraffin-embedded (FFPE) samples from primary tumors and/or paired metastases by means of Sanger Sequencing, Sequenom MassArray technology (Myriapod Colon status, Diatech Pharmacogenetics), or Ion Torrent PGM sequencing (SiRe next-generation sequencing panel).

\section{Pathologic evaluation and IHC analysis}

Two experienced gastrointestinal pathologists, who were blinded to mutational status and patients' outcome, revised specific pathologic features of each sample.

Available primary and/or metastatic FFPE surgical samples were processed using the Galileo CK3500 Arrayer, a semiautomatic and computer-assisted tissue microarray platform. Four and three tissue cores ( $1 \mathrm{~mm}$ in diameter) were obtained from each primary and metastatic lesion, respectively. Small biopsy samples were processed separately. IHC stainings were automatically performed using the Bond Polymer Refine Detection kit (Leica Biosystems) in the BOND-MAX system (Leica Biosystems) on $4-\mu \mathrm{m}$-thick sections. Primary antibodies, dilutions, and scoring evaluation are available upon request.

DNA mismatch repair machinery deficient tumors (MMRd) were defined in the absence of nuclear immunostaining for one of the couples MLH1/PMS2 or MSH2/MSH6 in tumor cells (12).

Consensus molecular subgroups (CMS) were qualified according to Ten Hoorn and colleagues (13) by assessing four IHC markers (FRMD6, ZEB1, HTR2B, CDX2) in combination 
Schirripa et al.

Table 1. Baseline characteristics and major clinical parameters ${ }^{\mathrm{a}}$

\begin{tabular}{|c|c|c|c|c|c|c|c|c|c|}
\hline Characteristics & & $\begin{array}{l}\text { BRAF wt } \\
\text { TOT }=540 \mathrm{~N}(\%)\end{array}$ & $\begin{array}{l}\text { BRAF mut class } 1 \\
\text { TOT }=92 N(\%)\end{array}$ & $\begin{array}{l}\text { BRAF mut class } 2 \\
\text { TOT }=12 \mathrm{~N}(\%)\end{array}$ & $\begin{array}{l}\text { BRAF mut class } 3 \\
\text { TOT }=13 N(\%)\end{array}$ & $\boldsymbol{P}$ & $p^{b}$ & $p^{c}$ & $P^{d}$ \\
\hline \multirow[t]{2}{*}{ Sex } & Female & $200(37)$ & $45(49)$ & $6(50)$ & $6(46)$ & 0.0458 & 1.0000 & 1.0000 & 1.0000 \\
\hline & Male & $340(63)$ & $47(51)$ & $6(50)$ & $7(54)$ & & & & \\
\hline Age & Median (range) & $62(21-91)$ & $69(35-85)$ & $60(45-89)$ & $56(45-83)$ & 0.004 & 0.115 & 0.759 & 0.251 \\
\hline \multirow[t]{2}{*}{ Age } & $>70$ & $132(24)$ & $39(42)$ & $5(42)$ & $3(23)$ & 0.0007 & 0.4110 & 1.0000 & 0.2351 \\
\hline & $\leq 70$ & $408(76)$ & $53(58)$ & $7(58)$ & $10(77)$ & & & & \\
\hline \multirow[t]{3}{*}{ Baseline ECOG PS } & 0 & $373(69)$ & $64(70)$ & $6(55)$ & $11(85)$ & 0.8875 & 0.2506 & 1.0000 & 0.167 \\
\hline & 1 & $139(26)$ & $22(24)$ & $3(18)$ & $0(0)$ & & & & \\
\hline & $\geq 2$ & $28(5)$ & $6(6)$ & $3(27)$ & $2(15)$ & & & & \\
\hline \multirow[t]{2}{*}{ Primary tumor resected } & Yes & $314(58)$ & $75(82)$ & $9(75)$ & $10(77)$ & $<0.0001$ & 1.0000 & 0.6965 & 0.7097 \\
\hline & No & $226(42)$ & $17(18)$ & $3(25)$ & $3(23)$ & & & & \\
\hline \multirow[t]{4}{*}{ Primary tumor location } & Right & $137(26)$ & $73(79)$ & $4(33.3)$ & $0(0)$ & $<0.0001$ & 0.4666 & 0.1294 & 0.0028 \\
\hline & Left & $265(49)$ & $14(15)$ & $4(33.3)$ & $6(46)$ & & & & \\
\hline & Rectum & $136(25)$ & $5(5)$ & $4(33.3)$ & $7(54)$ & & & & \\
\hline & NA & 2 & 0 & 0 & 0 & & & & \\
\hline \multirow[t]{2}{*}{ Presentation of mets } & Synchronous & $383(71)$ & $60(65)$ & $7(58)$ & $7(54)$ & 0.3401 & 1.0000 & 0.7508 & 0.5395 \\
\hline & Metachronous & $157(29)$ & $32(35)$ & $5(42)$ & $6(46)$ & & & & \\
\hline \multirow[t]{3}{*}{ Mucinous histology } & Yes & $34(7)$ & $26(31)$ & $2(17)$ & $3(23)$ & $<0.0001$ & 1.0000 & 0.4993 & 1.0000 \\
\hline & No & $497(93)$ & $58(69)$ & $10(83)$ & $10(77)$ & & & & \\
\hline & NA & 9 & 8 & 0 & 0 & & & & \\
\hline \multirow[t]{3}{*}{ pT } & $1-2$ & $58(14)$ & $3(4)$ & $2(20)$ & $2(20)$ & 0.013 & 1.0000 & 0.0828 & 0.0828 \\
\hline & $3-4$ & $350(86)$ & $83(96)$ & $8(80)$ & $8(80)$ & & & & \\
\hline & NA & 132 & 6 & 2 & 3 & & & & \\
\hline \multirow[t]{3}{*}{$\mathrm{pN}$} & 0 & $104(26)$ & $14(17)$ & $4(40)$ & $5(56)$ & 0.0973 & 0.6563 & 0.0944 & 0.0159 \\
\hline & $\geq 1$ & $292(74)$ & $70(83)$ & $6(60)$ & $4(44)$ & & & & \\
\hline & NA & 144 & 8 & 2 & 4 & & & & \\
\hline \multirow[t]{2}{*}{ Metastatic sites, $n$} & 1 & $358(66)$ & $61(66)$ & $9(75)$ & $10(77)$ & 0.9203 & 1.0000 & 0.7467 & 0.5406 \\
\hline & $\geq 2$ & $182(34)$ & $31(34)$ & $3(25)$ & $3(23)$ & & & & \\
\hline
\end{tabular}

${ }^{a}$ At the time of first-line treatment start or, for candidates to BSC only, at the first visit for metastatic disease.

${ }^{\text {b}}$ Class 2 versus class 3.

${ }^{\mathrm{c}}$ Class 1 versus class 2.

${ }^{\mathrm{d}}$ Class 1 versus class 3.

with pan-cytokeratin (KER) to normalize results. Primary tumors and/or metastasis were then categorized into the three CMS classes (CMS1, CMS2/3, or CMS4) using the online classification tool (refs. 14, 15; Supplementary Fig. S1).

In $B R A F$ class 2 and 3 cases, histologic type and grading was revised according to the last updated WHO classification (16). Cytokeratin expression pattern was evaluated by CK7 and CK20 expression, while tumor-infiltrating lymphocytes by means of positive intratumoral CD3/CD8 elements. To stratify class 2 and 3 tumors according to Barras and colleagues (7) in BM1 and BM2 groups, an IHC profiling for CDK1, ATM, Phospho-AKT (Ser473), Cyclin D1, and Phospho-4E-BP1 (Thr70) expression was performed (Supplementary Fig. S2). According to the Barras' article, retained expression of ATM, activation of the AKT/4EB-P1 cascade (considering both the phosphorylated forms of AKT and 4E-BP1), low CDK1 expression, and high Cyclin D1 expression are BM1 markers. Because no a priori criteria have been defined, we exploratively categorized each tumor based on their IHC profiles as follows.

To stratify class 2 and 3 tumors according to Barras and colleagues in $\mathrm{BM} 1$ and $\mathrm{BM} 2$ groups, we exploratively categorized each tumor based on the presence/absence of these five markers: CDK1, ATM, Phospho-Akt (Ser473), Cyclin D1, and Phospho-4EBP1 (Thr70). Because BM1 is characterized by activation of PI3K/
mTOR/AKT pathway, while BM2 of cell-cycle pathway, we assigned samples to BM1 or BM2 based on the coherence of the following parameters. Overexpression of Phospho-Akt, Phospho4E-BP1, ATM, and Cyclin D1, and downregulation of CDK1 were consistent with a $\mathrm{BM} 1$ profile. On the other hand, $\mathrm{BM} 2$ cases were characterized by overexpression of CDK1 and downregulation of the remaining markers. A tumor was considered positive in ATM if $>10 \%$ of tumor cells were positive for nuclear ATM staining. The activation of the AKT/4E-BP1 cascade was defined in the presence of high expression levels of the phosphorylated forms of AKT and/ or 4E-BP1. High levels of Cyclin D1 and CDK1 expression were defined in the presence of at least $50 \%$ of positive cancer cells (Cyclin D1 in the nucleus, CDK1 both in the nucleus and cytoplasm). Samples with 4 or 5 coherent parameters were defined as BM1 or BM2, whereas tumors in which three out of five parameters were coherent with the hypothesis were defined as borderline BM1 or BM2. Tumors with only one or two parameters coherent with the original classification were defined as not evaluable.

\section{Statistical analysis}

Fisher exact test or $\chi^{2}$ test were used when appropriate to compare clinical, pathologic, and molecular features according to BRAF mutational status (BRAF wild-type vs. BRAF-mutant class 
Table 2. Sites of metastasis at diagnosis

\begin{tabular}{|c|c|c|c|c|c|c|c|c|c|}
\hline \multicolumn{2}{|c|}{ Sites of mets at diagnosis } & \multirow{2}{*}{$\begin{array}{l}\text { BRAF wt } \\
\text { TOT }=\mathbf{5 4 0 ~} \mathbf{N}(\%) \\
387(72)\end{array}$} & \multirow{2}{*}{$\begin{array}{l}\text { BRAF mut class } 1 \\
\text { TOT }=\mathbf{9 2} \mathbf{~} \mathbf{( \% )} \\
44(48)\end{array}$} & \multirow{2}{*}{$\begin{array}{l}\text { BRAF mut class } 2 \\
\text { TOT }=12 \mathbf{N}(\%) \\
8(67)\end{array}$} & \multirow{2}{*}{$\begin{array}{l}\text { BRAF mut class } 3 \\
\text { TOT }=13 \mathbf{N}(\%) \\
10(77)\end{array}$} & \multirow{2}{*}{$\begin{array}{l}\boldsymbol{P} \\
<0.0001\end{array}$} & \multirow{2}{*}{$\frac{\boldsymbol{P}^{\mathbf{a}}}{0.6728}$} & \multirow{2}{*}{$\frac{\boldsymbol{p}^{\mathbf{b}}}{0.3579}$} & \multirow{2}{*}{$\frac{\boldsymbol{p}^{\boldsymbol{c}}}{0.0740}$} \\
\hline Liver & Yes & & & & & & & & \\
\hline & No & $153(28)$ & $48(52)$ & $4(33)$ & $3(23)$ & & & & \\
\hline \multirow[t]{2}{*}{ Lung } & Yes & $104(19)$ & $15(16)$ & $2(18)$ & $2(15)$ & 0.6101 & 1.0000 & 1.0000 & 1.0000 \\
\hline & No & $436(81)$ & 77 (84) & $10(82)$ & $11(85)$ & & & & \\
\hline \multirow[t]{2}{*}{ Distant nodes } & Yes & $109(20)$ & $38(41)$ & $2(18)$ & $2(15)$ & $<0.0001$ & 1.0000 & 0.1234 & 0.1245 \\
\hline & No & $431(80)$ & $54(59)$ & $10(82)$ & $11(85)$ & & & & \\
\hline \multirow[t]{2}{*}{ Peritoneum } & Yes & $102(19)$ & $29(31)$ & $3(27)$ & $0(0)$ & 0.0098 & 0.0957 & 0.7510 & 0.0176 \\
\hline & No & $438(81)$ & $63(69)$ & $9(73)$ & $13(100)$ & & & & \\
\hline \multirow[t]{2}{*}{ Other } & Yes & $60(11)$ & $12(13)$ & $1(10)$ & $3(23)$ & 0.729 & 0.5930 & 1.0000 & 0.3928 \\
\hline & No & $480(89)$ & $80(87)$ & $11(90)$ & $10(77)$ & & & & \\
\hline
\end{tabular}

${ }^{a}$ Class 2 versus class 3.

${ }^{\mathrm{b}}$ Class 1 versus class 2 .

${ }^{\mathrm{C}}$ Class 1 versus class 3 .

1 vs. $B R A F$-mutant class 2 vs. $B R A F$-mutant class $3 ; B R A F$-mutant class 1 vs. BRAF-mutant class 2; BRAF-mutant class 1 vs. BRAFmutant class 3 and $B R A F$-mutant class 2 vs. BRAF-mutant class 3 ). OS was defined as the time from the diagnosis of metastatic disease to death due to any cause whereas progression-free survival (PFS) was calculated from the date of first-line systemic treatment start to the first observed progression or death due to any cause. OS and PFS analyses were performed according to the Kaplan-Meier method and survival curves were compared using the log-rank test. Statistical significance was set at $P=0.05$ for a bilateral test. All analyses were carried out by means of MedCalc Software.

\section{Results}

Patients' characteristics, clinical outcome, and treatments

Class 1, 2, and 3 BRAF mutated included 92, 12, and 13 patients, respectively. BRAF wild-type patients were 540. Female patients were $49 \%, 50 \%, 46 \%$, and $37 \%$ in the 4 groups, respec- tively. Median age was $69,60,56$, and 62 years $(P=0.004)$. Rightsided primary tumor occurred in $79 \%, 33.3 \%, 0 \%$, and $26 \%$ of cases $(P<0.001$; Table 1$)$.

Class 3 BRAF-mutated patients were more frequently left-sided ( $46 \%$ vs. $15 \%, P=0.003$ ), with no locoregional nodal involvement $(56 \%$ vs. $17 \%, P=0.016)$ and no peritoneal spread $(100 \%$ vs. $69 \%, P=0.018)$ compared with class 1 ones. No differences were observed comparing class 2 with class 1 or class 3 cases (Tables 1 and 2).

Median OS was 21.0 versus 23.4 versus 44.5 versus 42.2 months, in BRAF-mutated class 1, 2, 3, and BRAF wild-type, respectively $(P<0.0001)$. Hazard ratio (HR) for OS was 2.38 (95\% CI 1.61-3.54) for class 1 BRAF mutated, 1.90 (95\% CI 0.85 4.26) for class 2 BRAF mutated, and 0.93 (95\% CI 0.51-1.69) for class $3 B R A F$ mutated compared with $B R A F$ wild-type patients (Fig. 1).

Median PFS from the beginning of the first-line treatment was 7.3 versus 7.0 versus 13.8 versus 10.1 months, in the four groups, respectively $(P<0.0001)$. HR for PFS was $2.02(95 \% \mathrm{CI}$,
Figure 1.

Median OS according to $B R A F$ mutation classes.

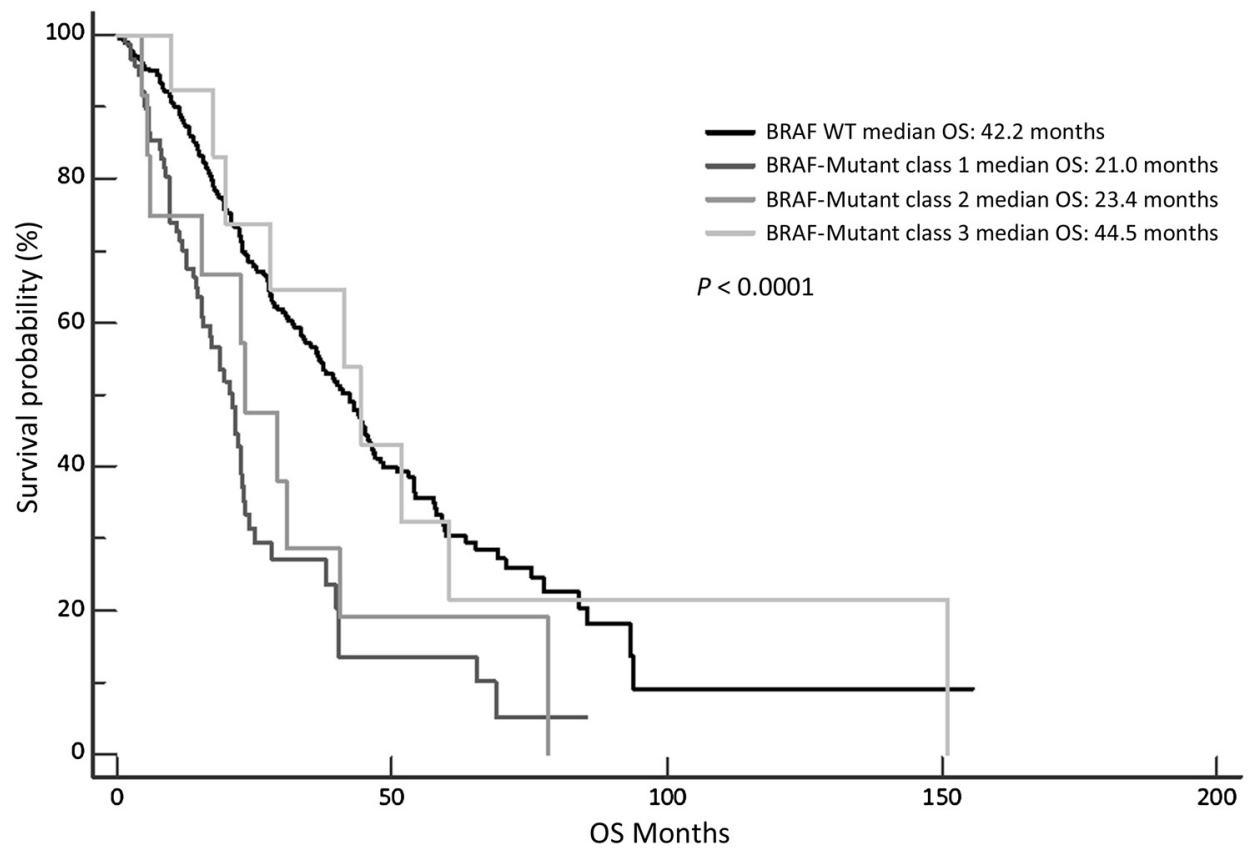




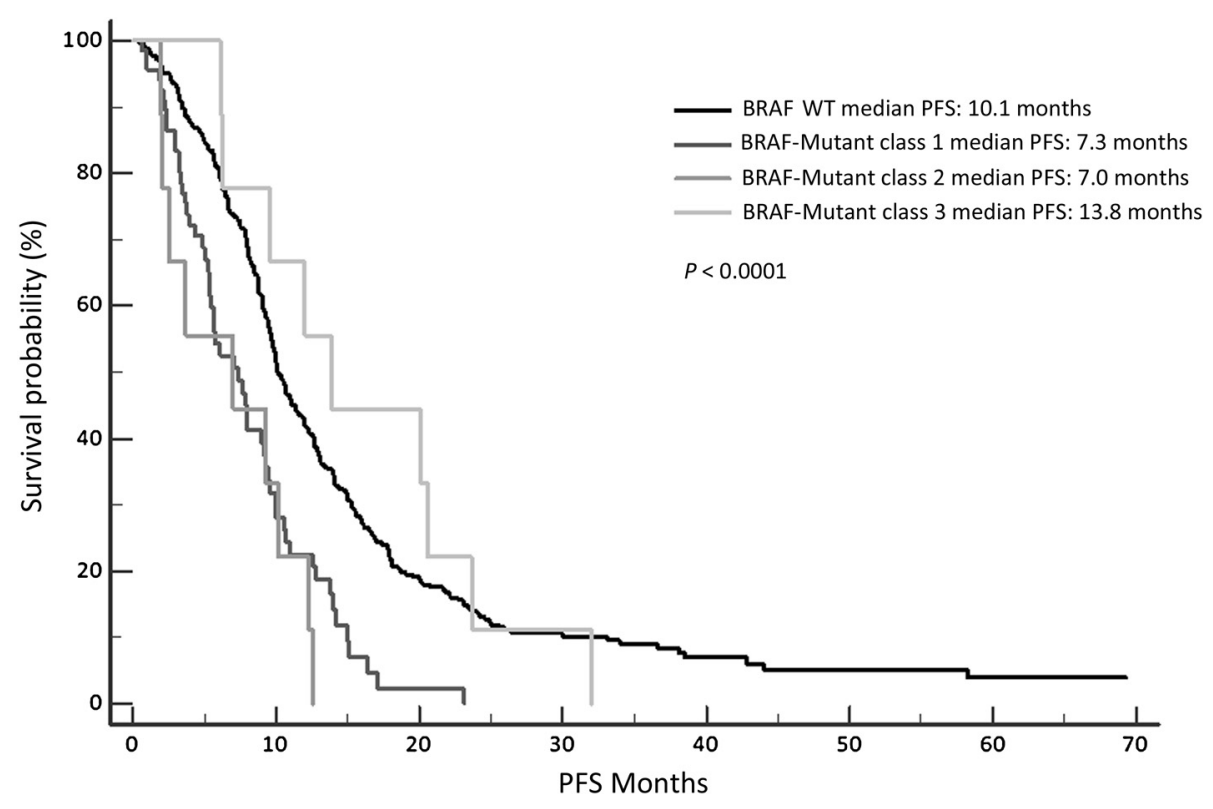

Figure 2.

Median PFS according to BRAF mutation classes.
1.39-2.94) for class 1 BRAF mutated, 2.49 (95\% CI, 0.92-6.74) for class 2 BRAF mutated, and 0.85 (95\% CI, 0.47-1.54) for class $3 B R A F$ mutated compared with $B R A F$ wild-type patients (Fig. 2).

In the group of BRAF wild-type patients, among 463 patients undergoing first-line chemotherapy, 49 received a monotherapy plus/minus a biologic agent, 288 received a doublet plus/minus a biologic agent, 124 received a three drugs combination plus/ minus a biologic agent, and 2 received other treatments. Overall, 292 of them received a bevacizumab-based treatment and 123 an anti-EGFR antibody.

Among 73 class 1 BRAF-mutated patients receiving first-line treatment, 7 underwent a monotherapy plus/minus a biologic agent, 43 received a doublet plus/minus a biologic agent, 14 received a three drugs combination plus/minus a biologic agent, and 9 received other treatments. Overall, 50 of them received a bevacizumab-based treatment and 7 received a treatment with an anti-EGFR antibody.

Nine of 12 class 2 BRAF-mutated patients received a first-line treatment. In particular, 2 underwent a monotherapy and 7 a doublet plus/minus a biologic agent. None of them received an anti-EGFR antibody.

Nine of 13 class 3 BRAF-mutated patients received a first-line treatment. In particular, 1 underwent a monotherapy, 5 a doublet plus/minus a biologic agent, 3 a three drugs combination plus/minus a biologic agent. Overall, 4 of them received a bevacizumab-based treatment and 5 received treatment with an anti-EGFR antibody.

\section{Mutational status and CMS in BRAF-mutant patients}

Among class 1 BRAF-mutated patients, adequate tissue specimens were available for 74 of 92 patients. In 40 of 74 cases, both primary and metastatic samples were available ( 9 cases had multiple metastatic sites available for IHC analysis). Overall, as indicated in Table 3, 39\% of class 1 tumors were defined as CMS1 (immune-like subtype), 44\% of patients as CMS 2-3 (epithelial-like subtype), and $17 \%$ of patients as CMS4 (mesenchymal-like subtype). Three cases were deemed as not evaluable due to lack of concordance between primary and metastatic samples.

Among class 2 BRAF-mutated patients, all tumors carried a BRAF p.K601E mutation. Of note, one tumor was characterized by a concomitant NRAS p.G12C mutation. Adequate tissue specimens were available for 11 of 12 patients. A total of 20 specimens were considered ( 8 primary and 12 metastatic); three cases had multiple metastatic sites available for IHC analysis and in three cases both primary and metastatic samples were available. All analyzed samples were categorized as CMS 2-3.

Among class $3 B R A F$-mutated patients, 2 tumors carried a $B R A F$ p.D594N mutation and 11 a BRAF p.D594G mutation. No other concomitant RAS mutation was identified. Adequate tissue

Table 3. Molecular stratification according to CMS

\begin{tabular}{|c|c|c|c|c|c|c|c|}
\hline & $\begin{array}{l}\text { BRAF mut class } 1 \\
\text { TOT }=74 \mathrm{~N}(\%)\end{array}$ & $\begin{array}{l}\text { BRAF mut class } 2 \\
\text { TOT }=11 \mathrm{~N}(\%)\end{array}$ & $\begin{array}{l}\text { BRAF mut class } 3 \\
\text { TOT }=10 \mathrm{~N}(\%)\end{array}$ & $\boldsymbol{P}$ & $P^{\mathbf{a}}$ & $p^{b}$ & $P^{c}$ \\
\hline CMS 1 - Immune-like & $28(39 \%)$ & $0(0 \%)$ & $0(0 \%)$ & \multirow{4}{*}{0.0146} & \multirow{4}{*}{1} & \multirow{4}{*}{0.0015} & \multirow{4}{*}{0.0009} \\
\hline CMS 2/3 - Epithelial-like & $31(44 \%)$ & $11(100 \%)$ & $10(100 \%)$ & & & & \\
\hline CMS 4 - Mesenchymal-like & $12(17 \%)$ & $0(0 \%)$ & $0(0 \%)$ & & & & \\
\hline NA & 3 & 0 & 0 & & & & \\
\hline
\end{tabular}

${ }^{a}$ Class 2 versus class 3 .

${ }^{\mathrm{b}}$ Class 1 versus class 2 .

${ }^{\mathrm{c} C l a s s} 1$ versus class 3. 
Table 4. Histopathologic features, tumor-infiltrating lymphocyte immunophenotype, and BM1/BM2 classification in BRAF-mutated class 2 and 3 patients

\begin{tabular}{|c|c|c|c|}
\hline & $\begin{array}{l}\text { BRAF mut class } 2 \\
\text { TOT }=11 N(\%)\end{array}$ & $\begin{array}{l}\text { BRAF mut class } 3 \\
\text { TOT }=10 N(\%)\end{array}$ & $\boldsymbol{P}$ \\
\hline \multicolumn{4}{|l|}{ Grading } \\
\hline Low & $6(54)$ & $7(70)$ & \multirow[t]{2}{*}{0.659} \\
\hline High & $5(46)$ & $3(30)$ & \\
\hline \multicolumn{4}{|l|}{ CK7 } \\
\hline Low (0-1) & $9(100)$ & $10(100)$ & \multirow[t]{3}{*}{1} \\
\hline High (2-3) & $0(0)$ & $0(0)$ & \\
\hline NE & 2 & 0 & \\
\hline \multicolumn{4}{|l|}{ CK20 } \\
\hline Low (0-1) & $2(29)$ & $4(40)$ & \multirow[t]{4}{*}{1} \\
\hline High (2-3) & $5(71)$ & $6(60)$ & \\
\hline Discordant & 2 & 0 & \\
\hline NE & 2 & 0 & \\
\hline \multicolumn{4}{|l|}{ CDX2 } \\
\hline Low (0-150) & $0(0)$ & $3(30)$ & \multirow[t]{4}{*}{0.211} \\
\hline High $(\geq 150)$ & $10(100)$ & $7(70)$ & \\
\hline Discordant & 1 & 0 & \\
\hline NE & 0 & 0 & \\
\hline \multicolumn{4}{|l|}{ CD3/CD8 } \\
\hline Low (0-20) & $5(56)$ & $10(100)$ & \multirow[t]{3}{*}{0.033} \\
\hline High $(>20)$ & $4(44)$ & $0(0)$ & \\
\hline NE & 2 & 0 & \\
\hline \multicolumn{4}{|c|}{ BM1/BM2 Classification } \\
\hline BM1 & $5(56)$ & $2(33)$ & \multirow[t]{3}{*}{0.608} \\
\hline BM2 & $4(44)$ & $4(67)$ & \\
\hline NA & 2 & 4 & \\
\hline
\end{tabular}

Abbreviations: NE, not evaluable; NA, not assessable.

specimens were available for 10 of 13 patients. Overall 18 samples were analyzed ( 8 primary and 10 metastatic); 3 cases had multiple metastatic sites available for IHC analysis and in 3 cases both primary and metastatic samples were available. All analyzed samples were categorized as CMS 2-3.

Pathologic features, lymphocyte infiltration, and BM1/BM2 classification in class 2 and $3 B R A F$-mutated patients

On the basis of the WHO classification, among class 2 BRAFmutated cases, 1 tumor had mucinous histology, 1 micropapillary histology, and 9 were not otherwise specified (NOS) adenocarcinomas. Tumor grade was high (i.e. grade 3 and 4) in 5 cases and low (i.e., grade 1 and 2) in the remaining six tumors. Intratumor phenotypic heterogeneity was evident only in a NOS adenocarcinoma, which showed intermingled areas of well-to-poor differentiation. No MMRd tumor was identified (Table 3 ). All cases showed a high CDX2 expression. In one case, metastatic samples showed a significant loss of CDX2 expression in comparison with the matched primary tumor; however, those metastatic lesions retained the same histologic grade and a strong CK20 positivity, as observed in primary samples. The analysis of cytokeratins expression and infiltrating lymphocytes evaluation was possible in 9 samples. Two tumors (29\%) were characterized by a low CK20 expression and two metastatic samples showed a significant loss in CK20 expression in comparison with their matched primary/ metastatic samples. All samples showed loss/low CK7 expres- sion, in particular, 3 cases (33.3\%) showed a faint (1+) CK7 immunoreactivity. Median CD3- and CD8-positive infiltrating lymphocytes per high power field were high in 4 cases (Table 4; Supplementary Fig. S3).

Among class 3 BRAF-mutated patients, based on the WHO classification, 2 tumors had micropapillary histology, 1 cribriform histology, and 7 were NOS adenocarcinomas. Intratumor phenotypic heterogeneity was evident in the 2 cases with micropapillary histology, which showed areas of poorly differentiated NOS histology. Tumor grade was high in 3 tumors and low in the remaining 7. As observed in class 2 lesions, no MMRd tumor was identified. Three cases (30\%) showed a low expression of CDX2. Four tumors (40\%) showed a low CK20 expression, all samples showed loss/low CK7 expression, whereas 2 samples showed a faint CK7 immunoreactivity. No significant intratumor heterogeneity for CMS, CDX2, and cytokeratins expression was observed in the 3 cases with multiple metastatic biopsies, nor among matched primary and metastatic samples. Median CD3- and CD8-positive infiltrating lymphocytes per high-power field were low in all cases (Table 4; Supplementary Fig. S3).

Overall no differences were observed among class 2 and 3 cases, with the exception of a higher median CD3- and CD8-positive lymphocytes infiltration in class 2 BRAF-mutated samples $(P=0.033$; Table 4$)$.

In class 2 BRAF-mutated tumors, 6 cases were positive for ATM, 7 showed an activation in AKT/4E-BP1, 2 were characterized by high levels of CDK1, and 3 by high levels of Cyclin D1. Overall, 5 cases were classified as BM1 (56\%), 4 as BM2 $(44 \%)$, whereas 2 tumors were not classifiable according to BM status (Table 4).

In class 3 BRAF-mutated tumors, 4 cases were positive for ATM, 5 showed an activation in AKT/4E-BP1, 5 were characterized by high levels of CDK1, and 5 by high levels of Cyclin D1. Overall, 2 cases were classified as BM1 (33\%), 4 as BM2 (67\%), whereas 4 tumors were not classifiable according to BM status (Table 4).

\section{Discussion}

Several clinical and pathologic descriptions of class $1 B R A F-$ mutated mCRC patients (i.e. V600E) have been published indicating specific features and overall poor life expectancy $(5,6)$. Our work characterized from a clinical, prognostic, and biological perspective the complete panel of known BRAF mutations according to functional classes, showing specific features for class 2 $B R A F$-mutated mCRC that have never been so extensively reported before, mainly due to their rarity. Moreover, we confirmed previous findings on class 1 and class 3 BRAF mutated $(8,10)$ and cross compared all the categories.

Looking at OS and PFS data, we observed that class 2 BRAFmutated patients have worse prognosis compared with class 3 and wild-type patients, and class 1-class 2 BRAF-mutated patients share similar poor median OS and PFS.

From a practical point of view, the identification of new subgroups of patients with mCRC with specific and rare BRAF mutations underlines the importance of the extensive adoption of modern techniques such as mass spectrometry or NGS in the daily clinical assessment of patients with mCRC.

In this study, we applied the CMS classification to the 3 $B R A F$ classes adopting the practical and rapid IHC method proposed by Trinh and colleagues (15), which allow to 
distinguish CMS2-3 from CMS4 cases, analyzing four specific markers (CDX2, FRMD6, HTR2B, and ZEB1). We defined CMS1 on the basis of MMRd status, testing for lack of expression of PMS2 and/or MSH6. All class 2 and 3 patients were classified as CMS2-3 and no heterogeneity was observed when pairing primary and/or metastatic samples. So that, disease development and progression might not be dependent on immune related mechanisms and/or mesenchymal-related pathways. Looking at class 1 BRAF-mutated patients, a wider heterogeneity has been detected since CMS1, 2, 3, and 4 occurred, respectively in $39 \%, 44 \%$, and $17 \%$ of cases. Among this group, such classification might provide further helpful information for patients' stratification and treatment decision making and would need specific studies. Recently Sveen and colleagues (17), investigated drug sensitivity according to CMS classification in preclinical models showing that CMS2 cell lines and PDX are more sensitive to EGFR and HER2 inhibition compared with other CMS subgroups. Unfortunately, IHC evaluation of CMS does not allow distinguishing CMS2 and 3 patients, so that to this extent further studies are needed.

To better characterize differences among class 2 and 3 patients, we performed a deep IHC characterization. CK7 and CK20 profiling revealed low rate of heterogeneity when looking at primaries and paired metastases and none of our cases showed a complete inversion of the CK7 and CK20 expression, already described elsewhere in some class 1 BRAF-mutated cases $(18,19)$.

Loss of CDX2 expression has been proposed as a negative prognostic feature in patients with mCRC and it is frequently associated with ${ }^{V 6 O O E} B R A F$ mutation and MMRd (18-20). In our series, loss of CDX2 was observed among three class 3 $B R A F$-mutated cases. Because of the small numbers, we were not able to further speculate on the prognostic impact of such feature.

Of note, CD3 and CD8 infiltration, already known as a prognostic feature in colorectal cancer (21-23), was higher in class 2 BRAF-mutated compared with class 3 patients $(P=0.033)$. Such finding might contribute to explain the outcome differences observed in our series.

From a therapeutic point of view, we cannot drive any definitive conclusion on anti-EGFR sensitivity of BRAF class 2 and 3 patients. A recent case report described a class 2 BRAFmutated mCRC patient achieving a durable response after treatment with panitumumab single agent (24). In our series, among class 2 BRAF mutated, only 3 patients received an antiEGFR with one responder, among class 3 BRAF mutated 6 patients received an anti-EGFR with 4 responders. Although definitive conclusions cannot be drawn, taking into account the kinase signaling mechanisms in class 2 and 3 patients, it could be hypothesized that class 3 patients might derive some benefit from anti-EGFRs due to their impaired kinase activity and to their RAS dependency. On the other hand, no reliable and specific data are available regarding systemic treatments of class 2 and 3 BRAF-mutated patients.

International guidelines recommend as first-line treatment for $B R A F$ class 1 patients with good performance status and younger than 75 years a 4 -drug combination (the triplet FOLFOXIRI plus bevacizumab regimen; ref. 1). Many trials have been performed and/or are ongoing to identify targeted strategies able to block the hyperactivated ${ }^{V O O E} B R A F$ signals. Disappointing results have been obtained with BRAF inhibitors alone (24-26) and currently, new therapeutic options are under evaluation for this subgroup of patients adopting $B R A F$ plus $M E K$ inhibitors together with anti-
EGFR mAbs (27). The recent stratification of class 1 BRAF-mutated patients into BM1 and BM2 upon gene expression laid the basis for a more tailored biologic approach in the development of targeted therapies. Specifically, BM1 subgroup is characterized by activation of KRAS/mTOR/AKT/4EBP1 pathway, whereas the BM2 had a deregulation in the cell cycle (7). In our study, we assigned samples to BM1 or BM2 category adopting a specific IHC expression analyses. Of note, 2 and 4 cases among class 2 and 3 patients, respectively, were not assigned to either BM1 or BM2 because they presented a peculiar IHC profile not properly matching with $\mathrm{BM} 1 / \mathrm{BM} 2$ categorization. One can hypothesize the presence of peculiar activation pathways for ${ }^{n o n-V 600 E} B R A F-$ mutated cases, however, we should also consider the intrinsic limitations of our results due to the application of a different technique (IHC instead of gene expression) and the limited sample size of our work.

Interestingly, in vitro sensitivity of class 2 and $3 B R A F$ mutations to MEK and BRAF inhibition has been proven in melanoma, and a small number of patients with these mutations showed responses to treatment with MEK inhibitors (28-31). The largest cohort of patients was collected by Boweyer and colleagues describing the antitumor activity of trametinib in 5 patients bearing the rare class 2 or class 3 BRAF mutations (32). Moreover, to elucidate these differences, functional studies were conducted in melanoma models showing that the activation of RAS and ERK pathways by these three classes through different mechanisms can explain their different sensitivity to therapeutic inhibitors: class 1 is sensitive to RAF "monomer" inhibitors (i.e. vemurafenib), class 2 is resistant to vemurafenib and could be sensitive to novel RAS dimer inhibitors (i.e. LY3009120) or MEK inhibitors (i.e. trametinib), class 3 is potentially sensitive to RTKs inhibitors (i.e., dasatinib; refs. 3, 5, 9, 11). Further studies are needed to clarify such mechanisms in colorectal cancer models and patients in the next future.

In conclusion, our study extensively described for the first time the ${ }^{\text {non-V6OOE }}$ BRAF mutations as two different subtypes of rare mCRC with specific clinical and prognostic and pathologic features that might be taken into account when planning new basic research initiatives and innovative clinical trials in this setting.

\section{Disclosure of Potential Conflicts of Interest}

S. Lonardi reports receiving speakers bureau honoraria from Lilly, Roche, and Bristol-Myers Squibb, and is a consultant/advisory board member for Amgen, Bayer, Merck, and Lilly. S. Corallo reports receiving speakers bureau honoraria from Pierre Fabre. F. Pietrantonio reports receiving speakers bureau honoraria from Amgen, Roche, Merck Serono, Eli Lilly, Bayer, Servier, and Sanofi. A.P. Dei Tos is a consultant/advisory board member for Lilly, Roche, PharmaMar, and Pfizer. V. Zagonel is a consultant/advisory board member for Bristol-Myers Squibb, Bayer, Roche, Novartis, Astellas, Pfizer, Jansen, Merck Serono, and Celgene. F. Loupakis is a consultant/advisory board member for Roche, Genentech, Bayer, and Amgen. No potential conflicts of interest were disclosed by the other authors.

\section{Authors' Contributions}

Conception and design: M. Schirripa, F. Gelsomino, F. Loupakis, M. Fassan Development of methodology: M. Schirripa, G. Fontanini, F. Loupakis, M. Fassan

Acquisition of data (provided animals, acquired and managed patients, provided facilities, etc.): M. Schirripa, S. Lonardi, N. Pella, M.S. Pino, F. Urbano, C. Antoniotti, C. Cremolini, S. Corallo, F. Pietrantonio, F. Gelsomino, S. Cascinu, A. Orlandi, U. Malapelle, M. Rugge, C. Mescoli, 
S. Lazzi, G. Lanza, A.P. Dei Tos, G. De Maglio, M. Martini, F. Bergamo, V. Zagonel, F. Loupakis, M. Fassan

Analysis and interpretation of data (e.g., statistical analysis, biostatistics, computational analysis): M. Schirripa, P. Biason, S. Lonardi, F. Gelsomino, G. Munari, M. Rugge, A.P. Dei Tos, V. Zagonel, F. Loupakis, M. Fassan

Writing, review, and/or revision of the manuscript: M. Schirripa, P. Biason S. Lonardi, N. Pella, C. Cremolini, F. Pietrantonio, F. Gelsomino, M. Rugge, C. Mescoli, G. Lanza, A.P. Dei Tos, F. Bergamo, V. Zagonel, F. Loupakis, M. Fassan

Administrative, technical, or material support (i.e., reporting or organizing data, constructing databases): P. Biason, F. Pietrantonio, S. Saggio, S. Lazzi, M. Fassan

\section{References}

1. Van Cutsem E, Cervantes A, Adam R, Sobrero A, Van Krieken JH, Aderka D, et al. ESMO consensus guidelines for the management of patients with metastatic colorectal cancer. Ann Oncol 2016;27:1386-22.

2. Clancy C, Burke JP, Kalady MF, Coffey JC. BRAF mutation is associated with distinct clinicopathological characteristics in colorectal cancer: a systematic review and meta-analysis. Colorectal Dis 2013;15:e711-18.

3. Ogino S, Brahmandam M, Cantor M, Namgyal C, Kawasaki T, Kirkner G, et al. Distinct molecular features of colorectal carcinoma with signet ring cell component and colorectal carcinoma with mucinous component. Mod Pathol 2006;19:59-68.

4. Naguib A, Mitrou PN, Gay LJ, Cooke JC, Luben RN, Ball RY, et al. Dietary, lifestyle and clinicopathological factors associated with BRAF and K-ras mutations arising in distinct subsets of colorectal cancers in the EPIC Norfolk study. BMC Cancer 2010;10:99.

5. Zheng G, Tseng LH, Chen G, Haley L, Illei P, Gocke CD, et al. Clinical detection and categorization of uncommon and concomitant mutations involving BRAF. BMC Cancer 2015; 15:779.

6. Comprehensive molecular characterization of human colon and rectal cancer. Nature 2012;487:330-37.

7. Barras D, Missiaglia E, Wirapati P, Sieber OM, Jorissen RN, Love C, et al. BRAF V600E mutant colorectal cancer subtypes based on gene expression. Clin Cancer Res 2017;23:104-15.

8. Jones JC, Renfro LA, Al-Shamsi HO, Schrock AB, Rankin A, Zhang BY, et al Non-V600 BRAF mutations define a clinically distinct molecular subtype of metastatic colorectal cancer. J Clin Oncol 2017;35:2624-30.

9. Yao Z, Yaeger R, Rodrik-Outmezguine VS, Tao A, Torres NM, Chang MT, et al. Tumours with class 3 BRAF mutants are sensitive to the inhibition of activated RAS. Nature 2017;548:234-38.

10. Cremolini C, Di Bartolomeo M, Amatu A, Antoniotti C, Moretto R, Berenato R, et al. BRAF codons 594 and 596 mutations identify a new molecular subtype of metastatic colorectal cancer at favorable prognosis. Ann Oncol 2015;26:2092-97.

11. Karoulia Z, Gavathiotis E, Poulikakos PI. New perspectives for targeting RAF kinase in human cancer. Nat Rev Cancer 2017;17:676-91.

12. Remo A, Fassan M, Lanza G. Immunohistochemical evaluation of mismatch repair proteins in colorectal carcinoma: the AIFEG/GIPAD proposal. Pathologica 2016;108:104-09.

13. Ten Hoorn S, Trinh A, De Jong J, Koens L, Vermeulen L. Classification of colorectal cancer in molecular subtypes by immunohistochemistry. Methods Mol Biol 2018;1765:179-91.

14. Colorectal Cancer Subtypes by IHC. Available from: https://crcclassifier. shinyapps.io/appTesting

15. Trinh A, Trumpi K, De Sousa E Melo F, Wang X, de Jong JH, et al. Practical and robust identification of molecular subtypes in colorectal cancer by immunohistochemistry. Clin Cancer Res 2017;23:387-98.

16. Bosman FT, Hruban RH, Theise ND. WHO classification of tumours of the digestive system. 4th ed. Lyon, France: IARC Press; 2010.

17. Sveen A, Bruun J, Eide PW, Eilertsen IA, Ramirez L, Murumägi A, et al Colorectal cancer consensus molecular subtypes translated to preclinical models uncover potentially targetable cancer cell dependencies. Clin Cancer Res 2018;24:794-06.

18. Kim JH, Rhee YY, Bae JM, Cho NY, Kang GH. Loss of CDX2/CK20 expression is associated with poorly differentiated carcinoma, the CpG
Study supervision: M. Schirripa, S. Lonardi, M. Rugge, S. Lazzi, F. Loupakis, M. Fassan

Other (histology): L. Reggiani Bonetti

\section{Acknowledgments}

This work was supported by a grant from Regione Veneto (RP-201400000395)

Received January 29, 2019; revised February 26, 2019; accepted April 3, 2019; published first April 9, 2019

island methylator phenotype, and adverse prognosis in microsatelliteunstable colorectal cancer. Am J Surg Pathol 2013;37:1532-41.

19. Landau MS, Kuan SF, Chiosea S, Pai RK. BRAF-mutated microsatellite stable colorectal carcinoma: an aggressive adenocarcinoma with reduced CDX2 and increased cytokeratin 7immunohistochemical expression. Hum Pathol 2014;45:1704-12.

20. Bruun J, Sveen A, Barros R, Eide PW, Eilertsen I, Kolberg M, et al. Prognostic, predictive, and pharmacogenomic assessments of CDX2 refine stratification of colorectal cancer. Mol Oncol 2018;12:1639-55.

21. Yoon HH, Shi Q, Heying EN, Muranyi A, Bredno J, Ough F, et al. Intertumoral heterogeneity of $\mathrm{CD}^{+}$and $\mathrm{CD} 8{ }^{+} \mathrm{T}$-Cell densities in the microen vironment of DNA mismatch-repair-deficient colon cancers: implications for prognosis. Clin Cancer Res 2018;25:125-133.

22. Pages F, Mlecnik B, Marliot F, Bindea G, Ou FS, Bifulco C, et al International validation of the consensus immunoscore for the classification of colon cancer: a prognostic and accuracy study. Lancet 2018 , 391:2128-39.

23. Allen WL, Dunne PD, McDade S, Scanlon E, Loughrey M, Coleman H, et al Transcriptional subtyping and CD8 immunohistochemistry identifies poor prognosis stage II/III colorectal cancer patients who benefit from adjuvant chemotherapy. JCO Precis Oncol 2018;2:1-15.

24. Corcoran RB, André T, Yoshito T, Bendell JC, Atreya CE, Schellens JHM, et al. Efficacy and circulating tumor DNA (ctDNA) analysis of the BRAF inhibitor dabrafenib (D), MEK inhibitor trametinib (T), and anti-EGFR antibody panitumumab (P) in patients (pts) with BRAF V600E- mutated (BRAFm) metastatic colorectal cancer (mCRC). Ann Oncol 2016;27: 4450 .

25. Hong D, Morris V, El Osta B, Sorokin AV, Janku F, Fu S, et al. Phase Ib study of vemurafenib in combination with irinotecan and cetuximab in patients with BRAF-mutated metastatic colorectal cancer and advanced cancers. J Clin Oncol 32:15s, 2015 (suppl; abstr 3511).

26. Corcoran RB, Atreya CE, Falchook GS, Kwak EL, Ryan DP, Bendell JC, et al. Combined BRAF and MEK Inhibition with dabrafenib and trametinib in BRAF V600-mutant colorectal cancer. J Clin Oncol 2015;33:4023-31.

27. Gong J, Cho M, Fakih M. RAS and BRAF in metastatic colorectal cancer management. J Gastrointest Oncol 2016;7:687-04.

28. Dahlman KB, Xia J, Hutchinson K, Ng C, Hucks D, Jia P, et al. BRAF(L597) mutations in melanoma are associated with sensitivity to MEK inhibitors. Cancer Discov 2012;2:791-97.

29. Richtig G, Aigelsreiter A, Kashofer K, Talakic E, Kupsa R, Schaider H, et al. Two case reports of rare BRAF mutations in exon 11 and exon 15 with discussion of potential treatment options. Case Rep Oncol 2016;9:543-46.

30. Kim KB, Kefford R, Pavlick AC, Infante JR, Ribas A, Sosman JA, et al. Phase II study of the MEK1/MEK2 inhibitor trametinib in patients with metastatic BRAF-mutant cutaneous melanoma previously treated with or without a BRAF inhibitor. J Clin Oncol 2013;31:482-89.

31. Marconcini R, Galli L, Antonuzzo A, Bursi S, Roncella C, Fontanini G, et al Metastatic BRAF K601E-mutated melanoma reaches complete response to MEK inhibitor trametinib administered for over 36 months. Exp Hematol Oncol 2017;6:6.

32. Bowyer SE, Rao AD, Lyle M, Sandhu S, Long GV, McArthur GA, et al. Activity of trametinib in K601E and L597Q BRAF mutation-positive metastatic melanoma. Melanoma Res 2014;24:504-08. 


\section{Clinical Cancer Research}

\section{Class 1, 2, and 3 BRAF-Mutated Metastatic Colorectal Cancer: A Detailed Clinical, Pathologic, and Molecular Characterization}

Marta Schirripa, Paola Biason, Sara Lonardi, et al.

Clin Cancer Res 2019;25:3954-3961. Published OnlineFirst April 9, 2019.

Updated version Access the most recent version of this article at: doi:10.1158/1078-0432.CCR-19-0311

Supplementary Access the most recent supplemental material at:

Material http://clincancerres.aacrjournals.org/content/suppl/2019/04/09/1078-0432.CCR-19-0311.DC1

Cited articles This article cites 29 articles, 6 of which you can access for free at:

http://clincancerres. aacrjournals.org/content/25/13/3954.full\#ref-list-1

E-mail alerts Sign up to receive free email-alerts related to this article or journal.

Reprints and To order reprints of this article or to subscribe to the journal, contact the AACR Publications Department a Subscriptions pubs@aacr.org.

Permissions To request permission to re-use all or part of this article, use this link http://clincancerres.aacrjournals.org/content/25/13/3954.

Click on "Request Permissions" which will take you to the Copyright Clearance Center's (CCC)

Rightslink site. 